\title{
CARACTERÍSTICAS CLÍNICAS DA MIGRÂNEA TRANSFORMADA
}

\author{
ABOUCH V. KRYMCHANTOWSKI*, JACKELINE S. S. BARBOSA*, \\ WILLIANS LORENZATTO*, CÉLIA CHEIM*, MARCOS ADRIANO*
}

\begin{abstract}
RESUMO - Grande parte dos pacientes com cefaléia diária atendidos em clínicas especializadas apresentam história de migrânea. Alguns autores referem-se a esta cefaléia como migrânea transformada, destacando sua menor intensidade e caraterísticas clínicas diferentes das migrâneas. O objetivo deste estudo foi avaliar a apresentação clínica da cefaléia diária em pacientes com história de migrânea antes de sua transformação em diária. Foram estudados retrospectivamente 215 pacientes. Observamos que porcentagem elevada dos pacientes com migrânea transformada apresentava dor em pressão ou peso, característica da cefaléia tipo tensional crônica. O caráter latejante ainda permaneceu como um achado importante, especialmente naqueles pacientes com ataques intermitentes de migrânea típica.
\end{abstract}

PALAVRAS-CHAVE: cefaléia crônica diária , migrânea transformada.

\section{Clinical features of transformed migraine}

ABSTRACT - Most daily headache patients seen in specialized clinics present a past history of migraine. Some authors refer to it as transformed migraine and emphasize its milder intensity and clinical characteristics different from migraine. The aim of this study was to evaluate the clinical presentation of the daily headache in patients with prior history of migraine. We studied retrospectively 215 patients. We observed that a significant percentage of the patients presenting the so-called transformed migraine, reported frontal and/or temporal bilateral pain and had pressure or tightening pain, which is a characteristic of chronic tension-type headache. It emphasizes the loss or changing of the standard migraine features. The pulsatile pain quality remained as an important feature, specially for those with intermittent typical migraine attacks.

KEY WORDS: chronic daily headache, transformed migraine.

Os pacientes com cefaléias diárias primárias frequentemente apresentam história de migrânea episódica (ME), que se inicia tipicamente nas segunda e terceira décadas de vida ${ }^{1,2}$. As crises de migrânea tornam-se progressivamente mais frequentes e as características típicas das migrâneas, tais como náuseas, vômitos, fotofobia, fonofobia e osmofobia, tornam-se menos intensas ou mesmo ausentes. Outras características de migrânea podem persistir, incluindo agravamento da cefaléia no período menstrual, fatores deflagradores identificáveis e localização unilateral. Nestes pacientes, a história familiar para migrânea está geralmente presente. Em função destas observações, autores como Mathew ${ }^{2}$ propuseram denominar esta cefaléia de migrânea transformada (MT). Em 1994, Silberstein e col ${ }^{1}$ propuseram que a MT fosse considerada uma subdivisão das migrâneas ${ }^{1,3}$ (Anexo 1). Para que este diagnóstico seja realizado, é necessária a presença de uma história pregressa de cefaléias preenchendo os critérios diagnósticos para migrânea, de acordo com a classificação da International Headache Society (IHS) ${ }^{4}$, e a presença de cefaléia com duração superior a quatro horas por dia, até 15 dias por

*Centro de Avaliação e Tratamento da Dor de Cabeça do Rio de Janeiro e Instituto de Neurologia Deolindo Couto da Universidade Federal do Rio de Janeiro (UFRJ). Aceite: 13-setembro-1999.

Dr Abouch V. Krymchantowski - Rua Siqueira Campos 43/1002 - 22031-070 Rio de Janeiro RJ - Brasil. Fax 021235 2688. Email: abouchkrym@openlink.com.br 
Anexo 1. Migrânea transformada: critérios propostos por Silberstein e col. ${ }^{1}$.

1.8 Migrânea transformada (MT)

A. História de migrânea episódica preenchendo quaisquer dos critérios da International Headache Society (IHS) 1.1 a 1.6.

B. Cefaléia diária ou quase diária ( $>15$ dias/mês) por $>1$ mês.

C. Média de duração da cefaléia de 4 horas/dia (se não tratada).

D. História de aumento da freqüência da cefaléia com diminuição da intensidade das características migranosas por no mínimo 3 meses.*

E. No mínimo um dos seguintes:

1. Não haver suspeita de quaisquer das patologias relacionadas nos grupos 5-11, da classificação da IHS.

2. Tal patologia é suspeitada, mas é excluída por investigações apropriadas.

3. Tal patologia está presente, mas a primeira crise de migrânea não corre em relação temporal íntima com essa patologia.

1.8.1 Migrânea transformada com uso excessivo de medicações sintomáticas (MS).

A. Preencher os critérios de 1.8 .

B. No mínimo um dos seguintes, por no mínimo 1 mês.

1. Uso de analgésicos simples (> 1000mg AAS/acetaminofen) $>5$ dias/semana.

2. Uso de combinações analgésicas (cafeína, medicações contendo barbitúricos) (> 3 comprimidos/ dia) $>3$ dias/semana.

3. Uso de narcóticos $>1$ comprimido/dia $>2$ dias/semana.

4. Uso de ergotamina ( $1 \mathrm{mg}$ VO ou 0,5mg VR) $>2$ dias/semana.

1.8.2 Migrânea transformada sem uso excessivo de MS.

A. Preenchendo os critérios de 1.8 .

B. Não satisfazer os critérios de uso excessivo de medicações de 1.8.1.

*Métodos devem ser desenvolvidos e validados para avaliar essas características; VO, via oral; VR, via retal.

OBS: Os grupos 5-11 referem-se a patologias como tumores, desordens intracranianas vasculares, entre outras.

mês. Como as características de apresentação destas cefaléias diárias podem ser bastante variáveis, unilaterais ou bilaterais, com intensidade leve, moderada ou intensa, de caráter pulsátil ou não, e, com ou sem características migranosas associadas, estes autores não consideraram necessário estabelecer características particulares para elas, já que em função de seu pleomorfismo, estes critérios pouco iriam acrescentar ${ }^{5,6}$. No entanto, é dada grande importância à história de transformação da cefaléia para progressivamente mais frequente e menos intensa, por no mínimo três meses, e, alguns pacientes com MT, continuam apresentando, de forma intermitente, ataques típicos de migrânea sobrepostos $^{1}$, o que, para Mathew ${ }^{2}$, representa diferentes estágios de transformação da migrânea.

Do ponto de vista clínico, a MT foi inicialmente descrita por MATHEW e col., em 19827, e definida como uma síndrome clínica com história natural característica. Analisando 80 pacientes com cefaléia diária há mais de 1 ano, estes autores observaram que 61 (76,25\%) apresentavam história prévia de migrânea episódica (ME) antes do desenvolvimento da dor diária. Alguns anos mais tarde, em 1987, Mathew e col. ${ }^{8}$ estudaram uma série maior de 630 pacientes, encontrando 489 (77,71\%) com história prévia de ME, em 444 pacientes (90,8\%) de migrânea sem aura e 45 (9,2\%) de migrânea com aura. Estes pacientes começavam a apresentar ataques episódicos de migrânea 
com ou sem aura na adolescência ou na terceira década de vida, que iam se tornando progressivamente mais frequentes e entremeados a episódios de cefaléia mais leve. Em torno dos 30 a 40 anos, passavam a apresentar cefaléia diária ou quase diária com características migranosas menos exuberantes e características de cefaléia do tipo tensional mais frequentes. Muitas vezes os pacientes apresentavam crises típicas de migrânea sobrepostas à cefaléia diária de intensidade mais leve ${ }^{8}$.

O objetivo deste estudo foi avaliar a apresentação clínica de pacientes com cefaléia diária não paroxística há mais de 6 meses, com história prévia de migrânea e progressivo aumento da frequência da dor e/ou com ataques típicos de migrânea intermitentes, sobrepostos a cefaléia diária.

\section{MÉTODO}

Foram estudados retrospectivamente 215 pacientes, 158 mulheres e 57 homens, com idades entre 12 e 83 anos (média 35 anos), atendidos em uma clínica de cefaléias e apresentando cefaléia diária não paroxística há mais de 6 meses. Todos os pacientes apresentavam história prévia de migrânea com ou sem aura, de acordo com os critérios da IHS. As características clínicas da cefaléia na primeira consulta, bem como as características da cefaléia primária antes de se tornar diária, foram avaliadas durante a primeira visita pelo mesmo cefaliatra. Foram anotados a localização, qualidade e intensidade da dor atual, tempo de duração e características da fase inicial de cada ataque. Também foram anotados se a dor acordava o paciente a noite ou pela manhã e sintomas associados, além da presença ou não de ataques dolorosos intermitentes com características diversas, sobrepostos aos padrões da dor diária e sua apresentação clínica.

\section{RESULTADOS}

Dos 215 pacientes, $49(22,8 \%)$ referiam dor frontotemporal bilateral, $45(20,9 \%)$ hemicraniana não alternante, $33(15,3 \%)$ dor bitemporal, $26(12,1 \%)$ dor difusa, $19(8,8 \%)$ cefaléia frontotemporal unilateral alternante, $18(8,4 \%)$ cefaléia bifrontal, $16(7,4 \%)$ relatavam dor periorbital unilateral e $57(26,5 \%)$ apresentavam dor em outras localizações (parieto occipital bilateral em 10, temporal unilateral em 10 e vértex em 7 , entre outras menos frequentes).

A respeito da qualidade da dor, embora alguns pacientes tenham referido mais de uma qualidade de dor, $80(37,2 \%)$ tinham dor latejante, $78(36,2 \%)$ referiam dor em pressão, $37(17,2 \%)$ apresentavam associação de dor em pressão e latejante, $9(4,2 \%)$ dor em queimação, $8(3,7 \%)$ dor em queimação e latejante e $16(7,4 \%)$ pacientes referiam outros tipos de dor, tais como repuxamento e pontada. Todos os pacientes relatavam dor diária moderada e 87 (40,5\%) informavam apresentar ataques intermitentes de dor intensa pulsátil agravada com atividades rotineiras, bem como incapacidade para atividades habituais, náuseas (e/ou vômitos) e foto e fonofobias. A presença de fatores desencadeantes tais como período menstrual, perda de refeição e estresse, foi observada em 164 $(76,2 \%)$ pacientes.

O diagnóstico do quadro inicial, antes da evolução para cefaléia diária, foi migrânea sem aura em 167 pacientes, migrânea com aura e sem aura em 46, e migrânea com aura em 2 pacientes. Cento e trinta e nove pacientes $(64,6 \%)$ informavam sintomas relacionados ao sono tais como insônia, sono interrompido, cefaléia noturna ou ao despertar, e astenia. Setenta e nove pacientes $(36,7 \%)$, relataram dificuldades de concentração e fixação de leitura, agravamento da dor com os esforços intelectuais e de visão, e distúrbios de memória. O consumo diário de medicamentos sintomáticos (MS) foi observado em 191(88,8\%) pacientes.

\section{DISCUSSÃO}

Grande parte dos pacientes apresentando migrânea transformada relatou dor frontotemporal bilateral. Mathew e col. ${ }^{7}$ observaram que $58 \%$ dos pacientes de sua série, apresentavam cefaléia predominantemente unilateral de intensidade severa e 54\% dos pacientes referiam a presença de dor frontotemporal ou frontal. Nas cefaléias intercríticas leves, a localização era ipsilateral às de intensidade severa ou àquelas com características mais evidentes de migrânea. 
Porcentagem elevada dos pacientes apresentava dor em pressão ou peso, uma característica da cefaléia tipo tensional crônica, enfatizando assim a perda e/ou alteração do padrão migranoso. $\mathrm{O}$ caráter latejante ainda permaneceu como um achado importante, especialmente naqueles pacientes com ataques intermitentes de migrânea típica. Estes achados foram previamente descritos por Jevoux ${ }^{4}$, e Silberstein \& Lipton $^{5}$ que destacaram o pleomorfismo destas cefaléias, mas enfatizaram a possibilidade de se apresentarem sem as características típicas das migrâneas. A identificação de fatores deflagadores das crises em um percentual elevado de pacientes foi também observada na série pioneira de Mathew e col. ${ }^{7}$, na qual, $88 \%$ dos pacientes referiam a piora ou ressurgimento da dor quando em contato com tais fatores.

O consumo abusivo de medicamentos sintomáticos, muitas vezes até em caráter diário, demonstrado em $88,8 \%$ dos pacientes, assim como a presença de sintomas relacionados ao sono observada em $64,6 \%$ dos pacientes, foi referida respectivamente por $87,2 \%$ e 73,6\% dos pacientes estudados por Mathew ${ }^{8}$. Em 1993, Mathew ${ }^{2}$ ainda identificou dois grupos distintos de pacientes com MT, um deles com uso excessivo de MS, e outro sem o uso excessivo de MS. Saper ${ }^{9}$ também observou que a maior parte dos pacientes com cefaléia diária primária por mais de 1 ano, utilizam MS de forma abusiva o que Srikiatkhachorn \& Phanthurachinda ${ }^{10}$ confirmaram em mais da metade de 220 pacientes avaliados em um centro específico para cefaléias na Tailândia . No tocante a outros fenômenos associados, Mathew e $\mathrm{col}^{11}$ relataram a presença frequente de sintomas psicossomáticos e sintomas relacionados ao sono tais como irritabilidade, insônia, fragmentação do sono, hiperventilação e palpitações, semelhante ao observado na série de Srikiatkhachorn \& Phanthurachinda ${ }^{10}$.

Em conclusão, deve-se ressaltar a presença, também relatada por outros autores, de aspectos psiquiátricos que podem exercer papel importante na tendência ao abuso de analgésicos e de outros medicamentos sintomáticos. Esta co-morbidade deve ser também considerada em relação aos sintomas associados, relacionados ou não ao sono. Finalmente, os autores observam nos pacientes com migrânea episódica transformada em diária, a modificação dos padrões de apresentação clínica da dor, que perde características migranosas e passa a exibir aspectos das cefaléias do tipo tensional. Ressaltam ainda, que a frequente associação entre o consumo excessivo e regular de medicações sintomáticas e o desenvolvimento de cefaléia crônica diária, pode representar um fator causal que deve ser enfaticamente prevenido.

\section{REFERÊNCIAS}

1. Silberstein SD; Lipton RB; Solomon S; Mathew NT. Classification of daily and near-daily headaches: proposed revisions to the IHS criteria. Headache 1994;34:1-7.

2. Mathew NT. Transformed migraine. Cephalalgia 1993;13 (Suppl 12):78-83.

3. Headache Classification Committee of the International Headache Society. Classification and diagnostic criteria for headache disorders cranial neurolgias and facial pain. Cephalalgia 1988;8(Suppl 7):1-96.

4. Jevoux C. Cefaléia crônica diária primária: a propósito de 52 casos. (Dissertação de Mestrado, Universidade Federal Fluminense). Niterói, 1996.

5. Silberstein SD; Lipton RB. Chronic daily headache. In Goadsby P, Silberstein SD (eds). Headache. Blue Books of Neurology. Newton:Butterworth-Heinemann, 1997:201-226.

6. Mathew NT, Stubits E, Nigam MP. Transformation of episodic migraine into daily headache: analysis of factors. Headache 1982;22:66-68.

7. Mathew NT, Reuveni U, Perez F. Transformed or evolutive migraine. Headache 1987;27:102-106.

8. Mathew NT. Chronic refractory headache. Neurology 1993; 43 (Suppl 3):26-33.

9. Saper JR. Headache disorders: current concepts in treatment strategies. Littleton, Wright-PSG,1983:23.

10. Srikiatkhachorn A, Phanthurachinda K. Prevalence and clinical features of chronic daily headache in a headache clinic. Headache 1997;37:277-280.

11. Mathew NT, Kurman R, Perez F. Intractable chronic daily headache. A persistent neuro bio-behavioral disorder. Cephalalgia 1989;9 (Suppl 10):180-182. 